ANNALES

POLONICI MATHEMATICI

$97.3(2010)$

\title{
Nonlinear separable equations in linear spaces and commutative Leibniz algebras
}

\author{
by D. Przeworska-Rolewicz (Warszawa)
}

\begin{abstract}
We consider nonlinear equations in linear spaces and algebras which can be solved by a "separation of variables" obtained due to Algebraic Analysis. It is shown that the structures of linear spaces and commutative algebras (even if they are Leibniz algebras) are not rich enough for our purposes. Therefore, in order to generalize the method used for separable ordinary differential equations, we have to assume that in algebras under consideration there exist logarithmic mappings. Section 1 contains some basic notions and results of Algebraic Analysis. In Section 2 we consider equations in linear spaces. Section 3 contains results for commutative Leibniz algebras. In Section 4 basic notions and facts concerning logarithmic and antilogarithmic mappings are collected. Section 5 is devoted to separable nonlinear equations in commutative Leibniz algebras with logarithms.
\end{abstract}

Introduction. The purpose of the present paper is to find solutions of some nonlinear equations and commutative algebras by a "separation of variables" using the methods of Algebraic Analysis (cf. Przeworska-Rolewicz [PR1-6].

Recall that the classical theorem for ordinary differential equations with separated variables can be stated as follows:

Theorem 0.1 (cf. Triebel [T]). Suppose that $-\infty<a<b<+\infty$, $-\infty<c<d<+\infty, f_{1} \in C^{1}[a, b], f_{2} \in C^{1}[a, b], f_{2}(y) \neq 0$ for $y \in[a, b]$, $x_{0} \in[a, b], y_{0} \in[c, d]$. Then the unique solution of the equation

$$
y^{\prime}=f_{1}(x) f_{2}(y)
$$

with the initial condition

$$
y\left(x_{0}\right)=x_{0}
$$

can be calculated from the equation

$$
F(x, y)=0, \text { where } F(x, y)=F_{2}(y)-F_{1}(x),
$$

2010 Mathematics Subject Classification: 47J25, 47A05, 34A05.

Key words and phrases: algebra with unit, Leibniz condition, logarithmic mapping, antilogarithmic mapping, separation of variables, initial value problem, algebraic analysis. 


$$
F_{2}(y)=\int_{y_{0}}^{y} \frac{d v}{f_{2}(v}, \quad F_{1}(x)=\int_{x_{0}}^{x} f_{1}(u) d u .
$$

A basic example for this theorem is the following

EXAMPLE 0.1 . Consider in $C[a, 1], 0<a<1$, the separable equation

$$
\frac{d y}{d t}=\frac{y}{x}, \quad \text { i.e. } \quad \frac{d y}{y}=\frac{d x}{x} .
$$

Here we have: $f_{1}(x)=\frac{1}{x}, f_{2}(y)=y$ for $x, y \in C^{1}[a, 1]$. This implies $\ln y=$ $\ln x+\ln c=\ln (c x)$, where $c \in \mathbb{R} \backslash\{0\}$ is arbitrary. Then $y=c x$.

If we require condition (0.2) to be satisfied for $x_{0}, y_{0} \in[a, 1]$ then we get $y_{0}=c x_{0}$. If it is the case, then $c=x_{0} / y_{0}$. So $F_{1}(y)=\ln y-\ln y_{0}=\ln \left(y / y_{0}\right)$, $F_{1}(x)=\ln x-\ln x_{0}=\ln \left(x / x_{0}\right)$ and $F(x, y)=\ln \left(y / y_{0}\right)-\ln \left(x / x_{0}\right)$.

It will be shown that the structures of linear spaces and commutative algebras (even if they are Leibniz algebras, i.e. algebras with the Leibniz product rule) are not rich enough for our purposes. Therefore, in order to generalize the method used for separable differential equations (cf. Theorem 0.1 and Example 0.1, and Triebel [T]), we have to assume that in the Leibniz algebras under consideration there exist logarithms (cf. [PR3-6]).

Section 1 contains some basic notions and results of Algebraic Analysis (without proofs). In Section 2 we consider equations in linear spaces. Section 3 contains some results for commutative Leibniz algebras. In Section 4 basic notions and facts (without proofs) about logarithmic and antilogarithmic mappings are collected. Section 5 is devoted to separable nonlinear equations in commutative Leibniz algebras with logarithms.

It seems that the first attempt at a theory of ordinary and partial nonlinear differential equations was given in the book of Ritt (cf. [R1, 2]), where the main tool was the implicit function theorem.

1. Basic notions of algebraic analysis. We recall here the following notions and theorems (without proofs; cf. [PR1-4]).

Denote by $\mathbb{N}, \mathbb{N}_{0}, \mathbb{R}, \mathbb{C}, \mathbb{Z}, \mathbb{Q}$ the sets of positive integers, nonnegative integers, reals, complexes, integers and rational numbers, respectively and by $\mathbb{F}$ any field of scalars. Moreover, $\mathbb{F}[t]$ denotes the set of all polynomials in $t$ with coefficients in $\mathbb{F}$.

Let $X$ be a linear space (over a field of characteristic zero).

- $L(X)$ is the set of all linear operators with domains and ranges in $X$;

- $\operatorname{dom} A$ is the domain of $A \in L(X)$;

- $\operatorname{ker} A=\{x \in \operatorname{dom} A: A x=0\}$ is the kernel of $A \in L(X)$;

- $L_{0}(X)=\{A \in L(X): \operatorname{dom} A=X\}$;

- $I(X)$ is the set of all invertible elements in $X$; 
- $I_{n}(X)=\left\{x \in X: \exists_{y \in X} y^{n}=x\right\}(n \in \mathbb{N})$; if $x \in I_{n}(X)$ and $x=y^{n}$ then $y=x^{1 / n}$ is said to be an $n$th root of $x$.

An operator $D \in L(X)$ is said to be right invertible if there is an operator $R \in L_{0}(X)$ such that $R X \subset$ dom $D$ and $D R=I$, where $I$ denotes the identity operator. The operator $R$ is called a right inverse of $D$. By $R(X)$ we denote the set of all right invertible operators in $L(X)$. Let $D \in R(X)$. Let $\mathcal{R}_{D} \subset L_{0}(X)$ be the set of all right inverses for $D$, i.e. $D R=I$ whenever $R \in \mathcal{R}_{D}$. We have

$$
\operatorname{dom} D=R X \oplus \operatorname{ker} D \quad \text { for all } R \in \mathcal{R}_{D} .
$$

Elements of $\operatorname{ker} D$ are called constants, since by definition, $D z=0$ if and only if $z \in \operatorname{ker} D$. We point out that, in general, constants are different from scalars, since they are elements of the space $X$. If two right inverses commute with each other, then they are equal.

An element $y \in \operatorname{dom} D$ is said to be a primitive for $x \in X$ if $y=R x$ for some $R \in \mathcal{R}_{D}$. Indeed, by definition, $x=D R x=D y$. Again, by definition, all $x \in X$ have primitives. Let

$$
\mathcal{F}_{D}=\left\{F \in L_{0}(X): F^{2}=F, F X=\operatorname{ker} D \text { and } \exists_{R \in \mathcal{R}_{D}} F R=0\right\} .
$$

Any $F \in \mathcal{F}_{D}$ is said to be an initial operator for $D$ corresponding to $R$. One can prove that any projection $F^{\prime}$ onto ker $D$ is an initial operator for $D$ corresponding to a right inverse $R^{\prime}=R-F^{\prime} R$ for any $R \in \mathcal{R}_{D}$.

If two initial operators commute with each other, then they are equal. Thus this theory is essentially noncommutative. An operator $F$ is initial for $D$ if and only if there is an $R \in \mathcal{R}_{D}$ such that

$$
F=I-R D \quad \text { on } \operatorname{dom} D \text {. }
$$

Even more: Write $\mathcal{R}_{D}=\left\{R_{\gamma}\right\}_{\gamma \in \Gamma}$. Then, by (1.1), we conclude that $\mathcal{R}_{D}$ induces in a unique way a family $\mathcal{F}_{D}=\left\{F_{\gamma}\right\}_{\gamma \in \Gamma}$ of initial operators defined by $F_{\gamma}=I-R_{\gamma} D$ on $\operatorname{dom} D \quad(\gamma \in \Gamma)$. Formula (1.1) yields (by a two-lines induction) the Taylor Formula:

$$
I=\sum_{k=0}^{n} R^{n} F D^{n}+R^{n} D^{n} \quad \text { on } \operatorname{dom} D^{n} \quad(n \in \mathbb{N}) .
$$

It is enough to know one right inverse in order to determine all right inverses and all initial operators. Note that superposition (if it exists) of a finite number of right invertible operators is again a right invertible operator.

The equation $D x=y \quad(y \in X)$ has the general solution $x=R y+z$, where $R \in \mathcal{R}_{D}$ is arbitrarily fixed and $z \in \operatorname{ker} D$ is arbitrary. However, if we put an initial condition: $F x=x_{0}$, where $F \in \mathcal{F}_{D}$ and $x_{0} \in \operatorname{ker} D$, then this equation has the unique solution $x=R x+x_{0}$. 
If $T \in L(X)$ belongs to the set $\Lambda(X)$ of all left invertible operators, then $\operatorname{ker} T=\{0\}$. If $D$ is invertible, i.e. $D \in \mathcal{I}(X)=R(X) \cap \Lambda(X)$, then $\mathcal{F}_{D}=\{0\}$ and $\mathcal{R}_{D}=\left\{D^{-1}\right\}$.

If $P(t) \in \mathbb{F}[t]$ then all solutions of the equation

$$
P(D) x=y, \quad y \in X,
$$

can be obtained by decomposing the rational function $1 / P(t)$ into vulgar fractions. One can distinguish subspaces of $X$ with the property that all solutions of (1.3) belong to a subspace $Y$ whenever $y \in Y$ (cf. von Trotha [T], [PR2]).

If $X$ is an algebra over $\mathbb{F}$ and $D \in L(X)$ is such that $x, y \in \operatorname{dom} D$ implies $x y, y x \in \operatorname{dom} D$, then we shall write $D \in \mathbf{A}(X)$. The set of all commutative algebras belonging to $\mathbf{A}(X)$ will be denoted by $\mathrm{A}(X)$. If $D \in \mathbf{A}(X)$ then

$$
f_{D}(x, y)=D(x y)-c_{D}[x D y+(D x) y] \text { for } x, y \in \operatorname{dom} D,
$$

where $c_{D}$ is a scalar dependent on $D$ only. Clearly, $f_{D}$ is a bilinear form, which is symmetric when $X$ is commutative, i.e. when $D \in \mathrm{A}(X)$. This form is called the non-Leibniz component (cf. [PR1]). If $D \in \mathbf{A}(X)$ then the product differentiation rule in $X$ can be written as follows:

$$
D(x y)=c_{D}[x D y+(D x) y]+f_{D}(x, y) \quad \text { for } x, y \in \operatorname{dom} D .
$$

If $D \in \mathbf{A}(X)$ and if $D$ satisfies the Leibniz condition:

$$
D(x y)=x D y+(D x) y \quad \text { for } x, y \in \operatorname{dom} D,
$$

then $X$ is said to be a Leibniz algebra. This means that in Leibniz algebras $c_{D}=1$ and $f_{D}=0$. The Leibniz condition implies that $x y \in \operatorname{dom} D$ whenever $x, y \in \operatorname{dom} D$. If $X$ is a Leibniz algebra with unit $e$ then $e \in \operatorname{ker} D$, i.e. $D$ is not left invertible.

Non-Leibniz components for powers of $D \in \mathbf{A}(X)$ are determined by recurrence formulae (cf. [PR1], [PR3]).

Suppose that $D \in \mathbf{A}(X)$ and $p \neq 0$ is a fixed scalar. Then $p D \in \mathbf{A}(X)$ and $c_{p D}=c_{D}, f_{p D}=p f_{D}$.

If $D_{1}, D_{2} \in \mathbf{A}(X)$, the superposition $D=D_{1} D_{2}$ exists and $D_{1} D_{2} \in$ $\mathbf{A}(X)$, then

$$
c_{D_{1} D_{2}}=c_{D_{1}} c_{D_{2}}
$$

and for $x, y \in \operatorname{dom} D=\operatorname{dom} D_{1} \cap D_{2}$,

$$
f_{D_{1} D_{2}}(x, y)=f_{D_{1}}(x, y)+D_{1} f_{D_{2}}(x, y)++c_{D_{1}} c_{D_{2}}\left[\left(D_{1} x\right) D_{2} y+\left(D_{2} x\right) D_{1} y\right] \text {. }
$$

For higher powers of $D$ in Leibniz algebras, by an easy induction from (1.6) and the Leibniz condition, we obtain the Leibniz formula

$$
D^{n}(x y)=\sum_{k=0}^{n}\left(\begin{array}{l}
n \\
k
\end{array}\right)\left(D^{k} x\right) D^{n-k} y \quad \text { for } x, y \in \operatorname{dom} D^{n} \quad(n \in \mathbb{N}) .
$$


2. Equations in linear spaces. We begin with a theorem which at first sight may look rather artificial. However, it plays a role in our subsequent considerations.

TheOREM 2.1 (cf. [PR1]). Suppose that $X$ is a linear space over a field $\mathbb{F}$ of scalars (of characteristic zero), $D \in R(X)$, dim $\operatorname{ker} D \neq 0$ and $F$ is an initial operator for $D$ corresponding to $R \in \mathcal{R}_{D}$. Let $\left\{H_{x}\right\}_{x \in X}$ be a family of mappings of $X$ into itself (in general, nonlinear with respect to $x$ ). Then:

(i) Every solution $x \in \operatorname{dom} D$ of the equation

$$
D x=H_{x} y, \quad \text { where } y \in X \text { is given, }
$$

is a solution of the equation

$$
x-R H_{x} y=z, \quad \text { where } z \in \operatorname{ker} D \text { is arbitrary. }
$$

(ii) Conversely, if a solution $x$ of (2.2) belongs to $\operatorname{dom} D$ then it is a solution of (2.1).

(iii) A solution of (2.1) with the initial condition

$$
F x=x_{0}, \quad \text { where } x_{0} \in \operatorname{ker} D \text { is given, }
$$

satisfies the equation

$$
x-R H_{x} y=x_{0} .
$$

Proof. (i) If $x \in \operatorname{dom} D$ satisfies (2.1) then, by our assumptions,

$$
0=D x-H_{x} y=D x-D R H_{x}=D\left(x-R H_{x} y\right),
$$

which implies (2.2).

(ii) Conversely, if $x \in \operatorname{dom} D$ satisfies (2.2) then

$$
0=D z=D\left(x-R H_{x}\right)=D x-D R H_{x} y=D x-H_{x} y,
$$

i.e. $x$ satisfies $(2.1)$.

(iii) By (i), $x \in \operatorname{dom} D$ satisfies the initial value problem for (2.1) with condition (2.3) if it satisfies (2.2) with condition (2.3). Since, by definitions, $F R=0$ and $F z=z$ whenever $z \in \operatorname{ker} D$, we find

$$
z=F z=F\left(x-R H_{x} y\right)=F x-F R H_{x} y=x_{0},
$$

i.e. $x$ satisfies $(2.4)$.

Example 2.1. Let $\mathbb{F}=\mathbb{R}, X=C[0,1], D=\frac{d}{d t}, R=\int_{0}^{t},(F x)(t) \equiv c$, $c \in \mathbb{R}$, for $t \in[0,1], x \in X$. Then an ordinary differential equation with separable variables

$$
x^{\prime}(t)=a(t) x(t), \quad \text { where } H_{x}=a x, a \in X \text { is given, }
$$

is equivalent to a Volterra integral equation

$$
x(t)-\int_{0}^{t} a(s) x(s) d s=c, \text { where } c \in \mathbb{R} \text { is an arbitrary constant. }
$$


It is well-known that this equation has a unique solution for each $c \in \mathbb{R}$. On the other hand, $X=C[0,1]$ is an algebra with respect to pointwise multiplication of functions. Therefore, any solution $x \neq 0$ of (2.5) satisfies the equation

$$
\frac{x^{\prime}(t)}{x(t)}=a(t), \quad \text { i.e. } \quad \frac{d}{d t} \ln x(t)=a(t),
$$

which implies

$$
\ln x(t)=\int_{0}^{t} a(s) d s+\ln c=\ln \exp \int_{0}^{t} a(s) d s+\ln c=\ln \left[c \exp \int_{0}^{t} a(s) d s\right],
$$

where $c \in \mathbb{R} \backslash\{0\}$ is arbitrary. Finally, we conclude that

$$
x(t)=c \exp \int_{0}^{t} a(s) d s, \quad \text { where } c \in \mathbb{R} \backslash\{0\}
$$

(cf. also Triebel [T] and Example 0.1).

Example 2.2 (cf. PR1]). Suppose that $D \in R(X)$, dim ker $D \neq 0$, $R_{0}, \ldots, R_{M+N-1} \in \mathcal{R}_{D}$

$$
\begin{gathered}
Q(D)=\sum_{k=0}^{N-1} Q_{k} D^{k}, \quad \text { where } Q_{0}, \ldots, Q_{N-1} \in L_{0}(X), \\
Q^{o}=\sum_{k=1}^{N-1} Q_{0} R_{M+k} \ldots R_{M+N-1}
\end{gathered}
$$

and the operator $I+Q^{o}$ is invertible. Let $\left\{H_{x}\right\}_{x \in X}$ be as in Theorem 2.1. Then

$$
D_{1}=Q(D) D^{M} \in R(X), \quad R_{1}=R_{0} \ldots R_{M+N-1}\left(I+Q^{o}\right)^{-1} \in \mathcal{R}_{D},
$$

ker $D_{1}=\left\{z=\left(I-R_{1} D_{1}\right) x, x \in \operatorname{dom} D\right\}=$

$$
\begin{array}{r}
\left\{z=R_{0} \ldots R_{M+N-1}\left(I+Q^{o}\right)^{-1}\left(\sum_{m=0}^{N-1} Q_{m} \sum_{k=m+1}^{N-1} R_{m} \ldots R_{k-1} z_{m+k}+z_{M+m}\right)\right. \\
\left.+\sum_{k=0}^{N-1} R_{0} \ldots R_{k-1} z_{k}: z_{0}, \ldots, z_{M+N-1} \in \operatorname{ker} D\right\} .
\end{array}
$$

Therefore any $x \in \operatorname{dom} Q(D) D^{M}$ satisfies the equation

$$
Q(D) D^{M} x=H_{x} y, \quad \text { where } y \in X(M \geq 0)
$$

if and only if $x$ satisfies the equation

$$
x-R_{0} \ldots R_{M+N-1}\left(I+Q^{o}\right)^{-1} H_{x} y=z, \quad z \in \operatorname{ker} Q(D) D^{M} .
$$

This is a generalization of Theorem 2.1 for operators of order greater than 1 . 
EXAMPLE 2.3 (cf. [PR1]). Let all assumptions of Example 2.2 be satisfied and let $R_{0}, \ldots, R_{N-1}=R$. Then every solution $x$ of (2.10) belongs to $\operatorname{dom} Q(D) D^{M}$ and satisfies the equation

$$
x-R^{N+M}[Q(I, R)]^{-1} H_{x} y=z,
$$

where

$$
\begin{gathered}
z=R^{N+M}[Q(I, R)]^{-1}\left(\sum_{m=0}^{N-1} \sum_{k=m}^{N-1} R^{k-m} z_{k}\right) \\
+\sum_{k=0}^{M+n-1} R^{k} z_{k} \in \operatorname{ker} D^{M+N} \quad \text { whenever } \quad z_{0}, \ldots, z_{M+N-1} \in \operatorname{ker} D \\
Q(t, s)=\sum_{k=0}^{N} Q_{k} t_{N-k}^{k}, \quad Q(I, R)=I+Q^{o}
\end{gathered}
$$

and the operator $I+Q^{o}$ is invertible. Conversely, every solution of (2.12) belonging to dom $Q(D) D^{M}$ satisfies (2.10). A similar result can be obtained for the operator $Q^{M} Q(D)$.

EXAmple 2.4. Let $X$ be a linear space (over $\mathbb{F}$ ). Let $D \in R(X)$ and let $R \in \mathcal{R}_{D}$. Suppose that $a \in \operatorname{ker} D, b \in \mathbb{F} \backslash\{0\}, y \in X$ and the mapping $f$ of $X$ into itself (not necessarily linear) are given. By definitions, $D a=0$, $D(b x)=b D x$ whenever $x \in X$. Consider the equation

$$
D x=f(a+b x) y .
$$

Observe that here we have a particular case of (2.1) with $H_{x}=f(a+b x)$ for $x \in X$. Write $u=a+b x$. Then $D u=D(a+b x)=b D x$ and $D x=b^{-1} u$. Thus (2.15) may be rewritten as $b^{-1} D u=f(u) y$, i.e.

$$
D u=b f(u) y .
$$

Since $b R x=R(b x)$ whenever $b \in \operatorname{ker} D, x \in X$, we conclude that $u \in$ $\operatorname{dom} D=\operatorname{dom} X$ is a solution of (2.16) if and only if it is a solution of the equation

$$
u-B R[f(u) y]=z, \quad \text { where } z \in \operatorname{ker} D \text { is arbitrary. }
$$

This means that $x=b^{-1}(u-a)$ is a solution of (2.15) if and only if $u$ is a solution of (2.17) belonging to dom $D$.

Let $F$ be an initial operator for $D$ corresponding to $R$. By definition, $F R=0$ and $F z=z$ whenever $z \in \operatorname{ker} D$. Then for a given $x_{0} \in \operatorname{ker} D$ and the initial condition

$$
F x=x_{0}
$$


we find

$$
\begin{aligned}
z & =F z=F\{u-b R[f(u) y]\}=F u-b F R[f(u) y]=F(a+b x)=F a+b F x \\
& =a+b x_{0}
\end{aligned}
$$

i.e. equation (2.17) with condition (2.18) implies the equation

$$
u-b R[f(u) y]=x_{0} .
$$

Since $u=a+b x,(2.19)$ can be rewritten as

$$
a+b x-b R[f(a+b x) y]=x_{0} .
$$

We conclude that $x \in \operatorname{dom} D$ is a solution of the initial value problem (2.15), (2.17): $D x=f(a+b x) y, F x=x_{0}$ if and only if $x \in \operatorname{dom} D$ is a solution of $(2.20)$.

Clearly, the equations considered in Examples 2.2-2.4 are, in a sense, analogues (in linear spaces) of the classical ordinary differential equations with separable variables. However, in order to solve their resolving equations in closed form, we need richer structures.

3. Equations in commutative Leibniz algebras. Let a commutative algebra $X$ (over a field $\mathbb{F}$ of scalars) with $D \in R(X), e \in \operatorname{dom} D$ satisfy the Leibniz condition (1.5):

$$
D(x y)=x D y+y D x \quad \text { for } x, y \in \operatorname{dom} D .
$$

Then $X$ is a $D$-algebra, since $x y \in \operatorname{dom} D$ whenever $x, y \in \operatorname{dom} D$. The set of all such algebras will be denoted by $L(D)$. Recall that $e \in \operatorname{ker} D$ (cf. Example 6.11 in [PR1]), i.e. the unit $e$ is a constant. We shall use the following properties (cf. also [PR1]):

Suppose that $X \in L(D)$ and $F$ is an initial operator for $D$ corresponding to $R \in \mathcal{R}_{D}$. Write $g=R e\left({ }^{1}\right)$, Then $D g=D R e=e$. Hence, by an easy induction,

$$
D^{n} g=0 \quad(n \in \mathbb{N} \backslash\{1\}), \quad D g=e .
$$

Since $e \in \operatorname{ker} D$, we have $F e=e$. Since $F R=0$, we find $F g=F R e=0$. So that, if $F$ is multiplicative then (again by an easy induction)

$$
F g^{n}=(F g)^{n}=0 \quad(n \in \mathbb{N}) .
$$

( $\left.{ }^{1}\right)$ Elements of the form $g=R e$, where $R \in \mathcal{R}_{D}$, play the role of an argument, since in the case considered in Example 2.1 we have $g(t)=\int_{0}^{t} 1 d s=t$ for $t \in[0,1]$. 
By (3.3), we have

$$
F p(g)=p(0)=p_{0} e \quad \text { for } p(t) \in \mathbb{F}[t],
$$

$$
F w(g)=w(0)=\frac{p_{0}}{\tilde{p}_{0}} \quad \text { for } w(t) \in \mathbb{Q}[t], w=\frac{p}{\tilde{p}}, \tilde{p}_{0} \neq 0, \text { i.e. } \tilde{p}(0) \in I(X),
$$

where

$$
p(t)=\sum_{k=0}^{n} p_{k} t^{k} \in \mathbb{F}[t]
$$

Indeed, $p(0)=p_{0} e$ and

$$
F p(g)=F \sum_{k=0}^{n} p_{k} g^{k}=\sum_{k=0}^{N} F g^{k}=\sum_{k=0}^{n} p_{k}(F g)^{k}=p_{0} e=p(0)
$$

i.e. $p(0)$ is invertible if and only if $p_{0} \neq 0$.

Proposition 3.1. Suppose that $X \in L(D), F$ is a multiplicative initial operator for $D$ corresponding to $R \in \mathcal{R}_{D}$ and $g=R e$. Then $g \notin I(X)$.

Proof. Suppose that $g \in I(X)$. By (3.3) and (3.5), we have $F g^{-1}=$ $(F g)^{-1}$. However, $F g=0$.

ExAmple 3.1. Suppose that all conditions of Example 2.1 are satisfied. If we consider $X=C[0,1]$ as an algebra (over $\mathbb{R}$ ) with respect to pointwise multiplication then $X=L(d / d t)$, since the Leibniz condition holds:

$$
\begin{gathered}
(x y)^{\prime}=x y^{\prime}+y x^{\prime} \quad \text { for } x, y \in C^{1}[0,1], \quad \text { where } \\
D x=\frac{d}{d t} x=x^{\prime}, \quad \operatorname{dom} D=C^{1}[0,1] .
\end{gathered}
$$

Let $(F x)(t)=x(0)$ for $x \in X$. Then $g(t)=t$. Hence $g(0)=0$ and $g^{-1}$ does not exist.

ExAmple 3.2. Suppose that $X \in L(D), R \in \mathcal{R}, g=R e \in I(X)$ and $f$ is a mapping of $X$ into itself (in general, nonlinear). Consider the equation

$$
D x=f\left(g^{-1} x\right) \quad(x \in \operatorname{dom} D) .
$$

Write $x=g u$. Then $u=g^{-1}$ and, by the Leibniz condition, we have

$$
f(u)=f\left(g^{-1} x\right)=D x=D(g u)=u D g+g D u=u e+g D u=u+g D u,
$$
i.e.

$$
D u=h(u), \quad \text { where } \quad h(u)=g^{-1}[f(u)-u](u \in \operatorname{dom} D) .
$$

We conclude that (3.8) has a solution $x \in \operatorname{dom} D$ if and only if (3.9) (with separable variables) has a solution $u \in \operatorname{dom} D$. In that case, solutions of (3.8) are of the form $x=g u$. 
In the classical case equation (3.8) becomes the so-called homogeneous ordinary differential equation $y^{\prime}=f(y / x)$ (cf. [T], also Example 0.1).

Clearly, if $R_{1} \neq R, R_{1} \in \mathcal{R}_{D}$ then the corresponding initial operator $F_{1}=\left(I-R_{1} D\right) \neq F$ and, by definitions, we have $F_{1} R=-F R_{1}$. Hence, if $F_{1}$ is multiplicative and $F_{1} g=F_{1} R e \neq 0$ is invertible then the element $F_{1} g^{-1}=\left(F_{1} g\right)^{-1}$ is well-defined.

ExAmple 3.3. Suppose that $X, D, F, R$ satisfy all conditions of Example 3.2. Let $h$ be a mapping (in general, nonlinear) of $X$ into itself. Then the equation

$$
D x=h(x) y, \quad \text { where } y \in X \text { is given }(x \in \operatorname{dom} D),
$$

with separable variables is equivalent to the equation

$$
x-R[h(x) y]=z, \quad \text { where } z \in \operatorname{ker} D \text { is arbitrary. }
$$

The proof is similar to that given in Example 3.2 for (3.8), (3.9). However, in order to solve (3.10), we may take another way. Suppose that $h(x) \in I(\operatorname{dom} D)$ whenever $x \in \operatorname{dom} D$. Again we obtain a separable equation equivalent to (3.10):

$$
[h(x)]^{-1} D x=y .
$$

The element $h_{1}(x)=R\left\{[h(x)]^{-1} D x\right\}$ is a primitive for $[h(x)]^{-1} D x$, whenever $x \in \operatorname{dom} D$. Indeed, (3.12) implies that

$$
D h_{1}(x)=D R\left\{[h(x)]^{-1} D x\right\}=[h(x)]^{-1} D x=D R y,
$$

i.e. $D\left[h_{1}(x)-R y\right]=0$. Hence we obtain the equation

$$
h_{1}(x)=R y+z, \quad \text { where } z \in \operatorname{ker} D \text { is arbitrary. }
$$

If $h_{1}(x)$ is a one-to-one mapping then we conclude that

$$
x=h_{1}^{-1}(R y+z), \quad \text { where } z \in \operatorname{ker} D \text { is arbitrary. }
$$

Example 3.3 can be generalized as follows.

Proposition 3.2. Suppose that $X \in L(D), F$ is a multiplicative initial operator for $D$ corresponding to $R \in \mathcal{R}_{D}$ and $h$ is a mapping of $X$ into itself (in general, nonlinear) such that $h(x) \in I\left(\operatorname{dom} D^{n}\right)$ whenever $x \in \operatorname{dom} D^{n}$ $(n \in \mathbb{N})$. Then the equation

$$
D^{n} x=h(x) y, \quad \text { where } y \in X \text { is given }\left(x \in \operatorname{dom} D^{n}\right),
$$

is equivalent to the equation

$$
x-R^{n}[h(x) y]=z, \quad \text { where } z \in \operatorname{ker} D \text { is arbitrary. }
$$

Write

$$
h_{1}(x)=R^{n}\left\{[h(x)]^{-1} D^{n} x\right\} \quad \text { for } x \in \operatorname{dom} D^{n}(n \in \mathbb{N}) .
$$


If $h_{1}$ is a one-to-one mapping of $X$ into itself then (3.16) has all solutions of the form

$$
\begin{aligned}
x=h_{1}^{-1}\left(R^{n} y+z\right), \quad \text { where } & \sum_{k=0}^{n-1} R^{k} z_{k} \in \operatorname{ker} D^{n}, \\
& z_{0}, \ldots, z_{n-1} \in \operatorname{ker} D \text { are arbitrary, }
\end{aligned}
$$

Proof. As before, (3.15) may be rewritten as

$$
y=[h(x)]^{-1} D^{n} x=D^{n} R^{n}[h(x)]^{-1}=D^{n} h_{1}^{-1}(x),
$$

which implies (3.16).

COROLlary 3.1. Suppose that all assumptions of Proposition 3.1 are satisfied, $g=R e$ and $y=g^{m}$ where $m \in \mathbb{N}$ is fixed. Then all solutions of the equation

$$
D^{n} x=h(x) g^{m} \quad(m, n \in \mathbb{N})
$$

are of the form

$$
\begin{gathered}
x=h_{1}^{-1}\left(\frac{g^{n+m}}{(n+1) \ldots(n+m)}+z\right), \quad \text { where } z=\sum_{k=0}^{n-1} R^{k} z_{k} \in \operatorname{ker} D^{n}, \\
z_{0}, \ldots, z_{n-1} \in \operatorname{ker} D \text { are arbitrary. }
\end{gathered}
$$

Proof. By Proposition 3.1, equation (3.19) has all solutions of the form (3.18) with $y=g^{m}$. Recall that constants are not zero divisors, because $X$ is a Leibniz algebra. Since $F$ is multiplicative, we have

$$
R^{k} e=\frac{(R e)^{k}}{k !}=\frac{g^{k}}{k !} \quad(k \in \mathbb{N})
$$

(cf. von Trotha [vT] and [PR1]), Then for all $m, n \in \mathbb{N}$ we have

$$
\begin{aligned}
R^{n} y & =R^{n} g^{n}=R^{n}(R e)^{m}=R^{n} m ! R^{m} e=m ! R^{n+m} e \\
& =m ! \frac{g^{n+m}}{(n+m) !}=\frac{g^{n+m}}{(n+1) \ldots(n+m)},
\end{aligned}
$$

which yields (3.21).

Equations of the form (3.21) will be solved in Section 5 in another way.

Proposition 3.3. Suppose that $X \in L(D), R \in \mathcal{R}_{D}$ and $a \in X$.

(i) $x \in \operatorname{dom} D$ is a solution of the equation

$$
D x=a x+y, \quad y \in X \text { is given, }
$$

if and only if $x \in \operatorname{dom} D$ satisfies the equation

$$
x-R(a x)=R y+z, \quad \text { where } z \in \operatorname{ker} D \text { is arbitrary. }
$$


(ii) If $F$ is an initial operator for $D$ corresponding to $R$ and we have an initial value condition

$$
F x=x_{0}, \quad x_{0} \in \operatorname{ker} D \text { is given, }
$$

then a solution of the initial value problem (3.23), (3.24) (if it exists) satisfies the equation

$$
x-R(a x)=R y+x_{0} .
$$

(iii) If the operator I - Ra is invertible then equation (3.23) (hence also (3.22)) has all solutions of the form

$$
x=(I-R a)^{-1}(R y+z) \quad \text { where } z \in \operatorname{ker} D \text { is arbitrary }
$$

and the initial value problem $(3.22)$, $(3,24)$ has a unique solution

$$
x=(I-R a)^{-1}\left(R y=x_{0}\right)
$$

(cf. [PR1], [PR3]).

Proof. If $x$ satisfies (3.22) then $D R y=y=D x-a x=D x-D R(a x)=$ $D[x-R(a x)$, i.e. $D[x-R(a x)-y]=0$, which implies (3.23). Conversely, if $x$ satisfies (3.23) then $y=D(R y+z)=D\left[x-R\left(a x_{0}\right]=D x-D R(a x)=\right.$ $D x-a x$. If $I-R a$ is an invertible mapping then (3.23) immediately implies (3.26). If $F$ is an initial operator for $D$ corresponding to $R$ then, by (3.26), we have $z=F z=F[x-R(a x)-R y]=F x-F R(a x)-F R y]=F x=x_{0}$ (cf. Propositions 3.1, 3.2 and Corollary 3.1).

ExAmple 3.4. Suppose that $X \in L(D)$ and $R \in \mathcal{R}_{D}$. Recall that

$$
D x^{n}=n x^{n-1} D x \quad \text { whenever } n \in \mathbb{N}, x \in \operatorname{dom} D
$$

(cf. [PR1]). This formula holds also for negative integers, i.e.

$$
D x^{-n}=-n x^{-n-1} D x \quad \text { whenever } n \in \mathbb{N}, x \in \operatorname{dom} D \cap I(X)
$$

Indeed, $D x^{-1}=x^{-2} D x$. Then for $n \geq 2$ we have

$$
\begin{aligned}
D x^{-1} & =\left(D x^{-1}\right)^{n}=D\left(x^{-1}\right)^{n}=n\left(x^{-1}\right)^{n-1} D x^{-1}=-n x^{-n+1} x^{-2} D x \\
& =-n x^{-n-1} D x .
\end{aligned}
$$

Consider the equation

$$
D x=a x^{m}, \quad \text { where } a \in X, m \in \mathbb{N} .
$$

By (3.29), this equation can be rewritten as follows:

$$
D x^{-(m+1)}=-(m+1) x^{-m} x^{-m} D x=-(m+1) x^{-m} a x^{m}=-(m+1) a,
$$

which implies that

$$
x^{-(m+1)}=R[-(m+1) a]+z=z-(m+1) r a, \quad z \in \text { ker } D \text { arbitrary. }
$$


Suppose now that there is a $z \in \operatorname{ker} D$ such that $z-(m+1) R a$ is invertible. Then $x^{m+1}=[z-(m+1) R a]^{-1}$. If $[z-(m+1) R a]^{-1} \in I_{m+1}(X)$ then

$$
x=\left\{[z-(m+1) R a]^{-1}\right\}^{1 /(m+1)}=[z-(m+1) R a]^{-1 /(m+1)} .
$$

Similarly, in order to solve the equation

$$
D x=a x^{-m} \quad(m \in \mathbb{N})
$$

we have to rewrite (3.31) as follows:

$$
D x^{m+1}=(m+1) x^{m} D x=(m+1) x^{m} a x^{-m}=(m+1) a,
$$

which implies $x^{m+1}=(m+1) R a+z$, where $z \in$ ker $D$ is arbitrary. If there is a $z \in \operatorname{ker} D$ such that $(m+1) R a+z \in I_{m+1}(X)$ then

$$
x=[z+(m+1) R a]^{1 /(m+1)} .
$$

To summarize, we conclude that the equations $\left({ }^{2}\right)$

$$
D x=a x^{\mp} \quad(m \in \mathbb{N})
$$

have solutions of the form

$$
x=[z \pm(m+1) R a]^{ \pm 1 /(m+1)},
$$

respectively, if there are $z \in \operatorname{ker} D$ such that elements of the form (3.34) exist (cf. also Example 5.3).

\section{Algebras with logarithms. We start with}

Definition 4.1. For $D \in \mathrm{A}(X)$, define a multifunction $\Omega: \operatorname{dom} D \rightarrow$ $2^{\operatorname{dom} D}$ as follows:

$$
\Omega u=\{x \in \operatorname{dom} D: D u=u D x\} \quad \text { for } u \in \operatorname{dom} D .
$$

The equation

$$
D u=u D x \quad \text { for }(u, x) \in \operatorname{graph} \Omega
$$

is said to be the basic equation. Clearly,

$$
\Omega^{-1} x=\{u \in \operatorname{dom} D: D u=u D x\} \quad \text { for } x \in \operatorname{dom} D .
$$

The multifunction $\Omega$ is well-defined and $\operatorname{dom} \Omega \supset \operatorname{ker} D \backslash\{0\}$.

Suppose that $(u, x) \in$ graph $\Omega, L$ is a selector of $\Omega$ and $E$ is a selector of $\Omega^{-1}$. By definitions, $L u \in \operatorname{dom} \Omega^{-1}, E x \in \operatorname{dom} \Omega$ and the following equations are satisfied: $D u=u D L u, D E x=(E x) D x$.

Any invertible selector $L$ of $\Omega$ is said to be a logarithmic mapping and its inverse $E=L^{-1}$ is said to be an antilogarithmic mapping. By $G[\Omega]$ we denote the set of all pairs $(L, E)$, where $L$ is an invertible selector of $\Omega$ and

$\left({ }^{2}\right)$ For brevity, we write here and below $\pm n(n \in \mathbb{N})$ for two different equations, for $n \in \mathbb{N}$ and for $-n \in \mathbb{N}$. This means that, as a matter of fact, we consider two types of equations (cf. also Section 5). 
$E=L^{-1}$. For any $(u, x) \in \operatorname{dom} \Omega$ and $(L, E) \in G[\Omega]$ the elements $L u, E x$ are said to be the logarithm of $u$ and the antilogarithm of $x$, respectively. The multifunction $\Omega$ is examined in [PR3]. The assumption that $X$ is a commutative algebra is for simplicity only.

Clearly, by definition, for all $(L, E) \in G[\Omega],(u, x) \in$ graph $\Omega$ we have

$$
E L u=u, \quad L E x=x ; \quad D E x=(E x) D x, \quad D u=u D L u .
$$

The logarithm of zero is not defined. If $(L, E) \in G[\Omega]$ then $L(\operatorname{ker} D \backslash$ $\{0\}) \subset \operatorname{ker} D, E(\operatorname{ker} D) \subset \operatorname{ker} D$. In particular, $E(0) \in \operatorname{ker} D$.

If $D \in R(X)$ then logarithms and antilogarithms are uniquely determined up to a constant.

Let $D \in \mathrm{A}(X)$ and let $(L, E) \in G[\Omega]$. A logarithmic mapping $L$ is said to be of the exponential type if $L(u v)=L u+L v$ for $u, v \in \operatorname{dom} \Omega$. If $L$ is of the exponential type then $E(x+y)=(E x)(E y)$ for $x, y \in \operatorname{dom} \Omega^{-1}$. We have proved that a logarithmic mapping $L$ is of the exponential type if and only if $X$ is a Leibniz commutative algebra (cf. [PR3]). Moreover, Le $=0$, i.e. $E(0)=e$. In Leibniz commutative algebras with $D \in R(X)$ a necessary and sufficient conditions for $u \in \operatorname{dom} \Omega$ is that $u \in I(X)$ (cf. [PR3]).

By $\operatorname{Lg}(D)$ we denote the class of those commutative algebras with $D \in$ $R(X)$ and with unit $e \in \operatorname{dom} \Omega$ for which there exist invertible selectors of $\Omega$, i.e. there exist $(L, E) \in G[\Omega]$. By $\mathrm{L}(D)$ we denote the class of those commutative Leibniz algebras with unit $e \in \operatorname{dom} \Omega$ for which there exist invertible selectors of $\Omega$. By these definitions, $X \in \mathbf{L g}(D)$ is a Leibniz algebra if and only if $X \in \mathrm{L}(D)$ and $D \in R(X)$. This class will be denoted by $L(D)$. This means that $L(D)$ is the class of those commutative Leibniz algebras with $D \in R(X)$ and with unit $e \in \operatorname{dom} \Omega$ for which there exist invertible selectors of $\Omega$, i.e. there exist $(L, E) \in G[\Omega]$.

Let $F$ be an initial operator for $D$ corresponding to $R \in \mathcal{R}_{D}$. Let $m \in \mathbb{N}$. Let $X \in \mathbf{L g}(D)$. Let $(L, E) \in G[\Omega]$. If

$$
F D^{j} L=0 \quad \text { for } j=0,1, \ldots, m-1,
$$

then $(L, E)$ is said to be $m$-normalized by $R$ and we write $(L, E) \in G_{R, m}[\Omega]$ (cf. Taylor formula (1.2)).

If ker $D=\{0\}$ then either $X$ is not a Leibniz algebra or $X$ has no unit. Thus, by our definition, if $X \in L(D)$ then ker $D \neq\{0\}$, i.e. the operator $D$ is right invertible but not invertible.

Theorem 4.1. Suppose that $X \in L(D), F$ is an initial operator for $D$ corresponding to $R \in \mathcal{R}_{D},(L, E) \in G[\Omega]$ and $A$ is an algebra isomorphism of $X$. Let $D^{\prime}=A^{-1} D A$ and let $\Omega^{\prime}: \operatorname{dom} D^{\prime} \rightarrow 2^{\operatorname{dom} D^{\prime}}$ be defined as follows:

$$
\Omega^{\prime} u=\left\{x \in \operatorname{dom} D^{\prime}: D^{\prime} u=u D^{\prime} x\right\} \quad \text { for } u \in \operatorname{dom} D^{\prime} .
$$

Then there are $\left(L^{\prime}, E^{\prime}\right) \in G\left[\Omega^{\prime}\right]$ and $L^{\prime}=A^{-1} L A, E^{\prime}=A^{-1} E A$. 
5. Equations in Leibniz algebras with logarithms. We start with

Proposition 5.1. Suppose that $X \in L(D),(L, E) \in G[\Omega]$ and $g=R e$ for some $R \in \mathcal{R}_{D}$. Then $g \in I(X)$ and

$$
D L g=g^{-1} \text {. }
$$

Proof. By definitions, $g \in \operatorname{dom} \Omega^{-1}$. Since $X$ is a Leibniz algebra, this implies that $g \in I(X)$. Moreover, $g \in \operatorname{dom} \Omega \subset \operatorname{dom} D$. We therefore conclude that $D L g=g^{-1} D g=g^{-1} D R e=g^{-1} e=g^{-1}$, since the basic equation (4.1) is satisfied by $g$.

Proposition 5.2. Suppose that all assumptions of Proposition 5.1 are satisfied and $R a+z \in \operatorname{dom} \Omega^{-1}$ for some $a \in X$ and all $z \in \operatorname{ker} D$. Then all invertible solutions of the equation

$$
D x=a x
$$

are of the form

$$
x=z E R a, \quad \text { where } z \in \operatorname{ker} D \text { is arbitrary. }
$$

If $F$ is a multiplicative initial operator for $D$ corresponding to $R$ such that $F a \in I(X)$ then the initial value problem

$$
D x=a x, \quad F x=x_{0}, \quad \text { where } x_{0} \in \operatorname{dom} D \text { is given, }
$$

has the unique solution

$$
x=x_{0} E R a .
$$

Proof. By definition, if $x$ is a solution of (5.2) then $x \in I(X) \cap \operatorname{dom} D$. Hence $D L x=x^{-1} D x=a+D R a$, i.e. $D(L x-R a)=0$. This implies that $L x-R a=z^{\prime} \in \operatorname{ker} D$ ( $z^{\prime}$ is arbitrary). Write $z=E z^{\prime}$. Then we get $x=E L x=E\left(R a+z^{\prime}\right)=\left(E z^{\prime}\right)(E R a)=z E R a$, since the Leibniz condition holds. Now, we shall prove that

$$
E F=F E, \quad L F=F L .
$$

Indeed, by definitions, $E L=L E=I$. Write $A=F E$. Then $A L=F E L=F$ $=L E F=L A$, which implies $F E=A=E L A=E F$. In order to prove the second equality, write $B=F L$. Then $B E=F L E=F=E L F=E B$ and $F=E L F=E B=B E$, which implies $F L=B E L=B=L F$.

If $F x=x_{0} \in \operatorname{ker} D$ then $F^{z}=F x_{0}=x_{0}$ and, by (5.6) and the multiplicativity of $F$, we find (5.5).

An initial operator $F \in \mathcal{F}_{D}$ is said to be almost averaging if

$$
F(z x)=z F x \quad \text { whenever } x \in X, z \in \operatorname{ker} D .
$$

Clearly, every multiplicative initial operator $F$ is almost averaging for $F(z x)$ $=(F z)(F x)=z F x$, but not conversely, even if $\operatorname{dim} \operatorname{ker} D=1$ (cf. [PR1]).

COROllary 5.1. Proposition 5.2 holds for almost averaging $F$. 
Indeed, for all $z \in \operatorname{ker} D$, we have $x_{0}=F x=F(z E R a)=z F E R a=$ $z E F R a=z E(0)=z e=z$, i.e. $x$ is of the form (5.5).

Proposition 5.3. Suppose that $X \in L(D),(L, E) \in G[\Omega], \pm R a \in$ $\operatorname{dom} \Omega^{-1}$ for some $a \in X$ and $F$ is an almost averaging initial operator for some $R \in \mathcal{R}_{D}$. Then the equation

$$
D x=a x+y, \quad \text { where } y \in X \text { is given, }
$$

has all solutions of the form

$$
x=(E R a) R E(-R a)+z E R a, \quad \text { where } z \in \text { ker } D \text { is arbitrary. }
$$

Proof. We are looking for solutions of (5.8) which are of the form $x=u v$, where $v=E R a$ and $u$ is to be determined. By the Leibniz condition and our assumptions, we have $D v=D E R a=(E R a) D R a=a E R a=a v$, i.e. $v$ is a solution of (5.2). Then

$$
\begin{aligned}
y & =D x-a x=D(u v)-a u v=u D v+v D u-a u v=u v D u+v D u-a u v \\
& =u v a+v D u-a u v=v D u .
\end{aligned}
$$

Since $v=E R a \in I(X)$ and $v^{-1}=(E R a)^{-1}=E(-R a)$, we find $D u=v^{-1} y$ $=y E(-R a)$, i.e.

$$
u=R[y E R(-a)]+z, \quad \text { where } z \in \operatorname{ker} D \text { is arbitrary. }
$$

Then

$$
x=u v=(E R a)\{R[y E(-R a)]+z\}=(E R a) R[y E(-R a)]+z E R a,
$$

i.e. $x$ is of the form (5.9) (cf. also [PR3]).

COROLlary 5.2. Suppose that all assumptions of Proposition 5.3 are satisfied and $F$ is a multiplicative initial operator for $D$ corresponding to $R$. Then an initial value problem for (5.8) with the initial condition

$$
F x=x_{0}, \quad \text { where } x_{0} \in \operatorname{ker} D \text { is given, }
$$

has the unique solution

$$
x=(E R a) R[y E(-R a)]+x_{0} E R a .
$$

Proof. By our assumptions and (5.6), (5.9), we find $F v=F E R a=$ $E F R a=E(0)=e$, and $F u=F\{R[y E(-R a)]+z\}=F R[y E(-R a)]+F z$ $=z$. This implies that $x_{0}=F x=F(u v)=(F u)(F v)=e z=z$, which was to be proved.

Note 5.1. We have seen that (according to Proposition 3.1), in order to solve the equations in question in closed form, we had to assume that the operator $I-R a$ is invertible and to calculate its inverse. In several cases this way could be much more complicated than the use of logarithms and antilogarithms. Also some metric properties of the space and operators may be necessary. 
EXAmPLE 5.1 (Generalized Pearson equation). Suppose that all assumptions of Proposition 5.3 are satisfied, $g=R e$ and $a=w(g)$, where $w(t) \in$ $\mathbb{Q}[t]$. Consider the equation

$$
D x=w(g) x .
$$

By definitions, if $x \in I(X) \cap \operatorname{dom} D$ is a solution of (5.13) then $D L x=$ $x^{-1} D x=w(g)$, i.e. $L x=R w(g)+z^{\prime}$, where $z^{\prime} \in \operatorname{ker} D$ is arbitrary. Write $z=E z^{\prime}$. Then $\in \operatorname{ker} D$ and $x=E L x=E\left[R w(g)+z^{\prime}\right]=\left(E z^{\prime}\right) E R w(g)=$ $z E R w(g)$ (cf. also Example 3.4 for $m=-1$ ). Observe that again $R w(g)(t) \in$ $\mathbb{Q}[t]$. The equation

$$
D x=w(g) x+y, \quad \text { where } y \in X \text { is given, }
$$

has all solutions of the form

$$
x=[E R w(g)] R\{y E[-R w(g)]\}+z E R w(g), \quad 0 \neq z \in \text { ker } D \text { arbitrary. }
$$

EXAmple 5.2. Suppose that all assumptions of Proposition 5.3 are satisfied, $g=\operatorname{Re} \in I(X)$ and $a=e$. Then the equation

$$
D x=g^{-1} x L x
$$

has all solutions belonging to $\operatorname{dom} \Omega \cap \operatorname{dom} \Omega^{-1}$ of the form

$$
x=E\left(z E R g^{-1}\right), \quad \text { where } z \in \operatorname{ker} D \text { is arbitrary. }
$$

Indeed, by our assumptions, whenever $x \in \operatorname{dom} \Omega \cap \operatorname{dom} \Omega^{-1}$, (5.15) implies $D L x=x^{-1} D x=g^{-1} x$, i.e $L x=z E R g^{-1}$, where $z \in$ ker $D$ is arbitrary (cf. Proposition 5.3). Then $x=E L x=E\left(z E R g^{-1}\right)$ (cf. Example 5.1).

Suppose that $F$ is an almost averaging initial operator for $D$ corresponding to $R$. By (5.6), for all $z \in \operatorname{ker} D$ we have

$$
\begin{aligned}
F x & =F E\left(z E R g^{-1}\right)=E F\left(z E R g^{-1}\right)=E\left(z F E R g^{-1}\right)=E\left(z E F R g^{-1}\right. \\
& =E[z E(0)]=E(z e)=E z \in \operatorname{ker} D
\end{aligned}
$$

(cf. Corollary 5.1). Therefore the initial condition $F x=x_{0}$, where $x_{0} \in$ $\operatorname{ker} D$ is given, for (5.15) holds if and only if $z=L E z=L F x=L x_{0} \in$ ker $D$. In that case, the corresponding initial value problem has the unique solution $x=E\left[\left(L x_{0}\right) E R g^{-1}\right]$. Observe that $L g=R g^{-1}$. Hence (5.15) can be rewritten as $x=E\left(z E R g^{-1}\right)=E(z E L g)=E(z g)$, which implies that the unique solution of the initial value problem in question is $x=E\left(g L x_{0}\right)$.

EXAMPLE 5.3. Suppose that all assumptions of Proposition 5.3 are satisfied and $a$ is a mapping of $X$ into itself such that $a(x) \neq e$ whenever $x \in \operatorname{dom} \Omega$. Consider the equation

$$
D x=a(x) x L x .
$$

The case $a(x) \equiv e$ has been considered in Example 5.2. As in that example, we obtain the equalities $D L x=x^{-1} D x=a(x) L x$, which implies $x=$ 
$E L x=E\left\{R[a(x) L x]+L z^{\prime}\right\}=E\{z R[a(x) L x]\}$, where $z=L z^{\prime}, z^{\prime} \in \operatorname{ker} D$ is arbitrary. Then, by (5.6), we find

$$
\begin{aligned}
F x & =F E\{z R[a(x) L x]\}=E F\{z R[a(x) L x]\}=E\{z F R[a(x) L x) \\
& =E(0)=e .
\end{aligned}
$$

We conclude that a necessary condition for (5.17) to have a solution $x$ is that $F x=e$.

Theorem 5.1. Suppose that $X \in L(D),(L, E) \in G[\Omega], \pm R a \in \operatorname{dom} \Omega$ for some $a \in X$ and $R \in \mathcal{R}_{D}$ and $F$ is an almost averaging initial operator for $D$ corresponding to $R$. For a given $m \in \mathbb{N}$ write

$$
v^{ \pm}=z \mp(m+1) R a
$$

whenever there exist $z \in \operatorname{ker} D$ such that

$$
v^{ \pm} \in I_{m+1}(X) \cap \operatorname{dom} \Omega .
$$

If (5.19) is satisfied then the equations

$$
D x=a x^{\mp} m
$$

have solutions of the form

$$
x^{ \pm}=v^{ \pm 1 /(m+1)}=E\left[\mp \frac{1}{m+1} L v^{ \pm}\right],
$$

respectively (cf. footnote in Example 3.4).

Proof. Let $m \in \mathbb{N}$. Consider the equation $D x=a x^{m}$. By our assumptions, we have

$$
D x^{-(m+1)}=-(m+1) x^{-m} D x=-(m+1) x^{-m} a x^{m}=-(m+1) a,
$$

which implies $x^{-(m+1)}=v^{+}$, where $v^{+}$is determined by (5.18). This and condition (5.19) together imply that $x^{+}$is a solution we are looking for. A similar proof for $-m \in \mathbb{N}$.

COROLlary 5.3. Suppose that all assumptions of Theorem 5.1 and condition (5.19) are satisfied. Then an initial condition

$$
F x=x_{0}, \quad \text { where } x_{0} \in \operatorname{ker} D \text { is given, }
$$

holds if and only if

$$
x_{0}=E\left(\mp \frac{1}{m+1} L z\right)=z^{\mp 1 /(m+1)} \text {, i.e. } z=x_{0}^{\mp(m+1)}=E\left(\mp \frac{1}{m+1} L x_{0}\right) .
$$


Proof. By our assumptions, (5.6) and Theorem 5.1, we have

$$
\begin{gathered}
F v^{ \pm}=F[z \pm(m+1) R a]=F z \pm(m+1) F R a=z, \\
x_{0}=F x^{ \pm}=F E\left(\mp \frac{1}{m+1} L v^{ \pm}\right)=E F\left(\mp \frac{1}{m+1} L v^{ \pm}\right) \\
=E\left(\mp \frac{1}{m+1} F L v^{ \pm}\right)=E\left(\mp \frac{1}{m+1} L F v^{ \pm}\right)=E\left(\mp \frac{1}{m+1} L F v^{ \pm}\right) \\
=E\left(\mp \frac{1}{m+1} L z\right)=E L z^{\mp 1 /(m+)}=z^{\mp 1 /(m+1)},
\end{gathered}
$$

i.e.

$$
x_{0}=E L x_{0}=E[\mp(m+1) L z]=z^{\mp(m+1)} .
$$

COROLlary 5.4. Suppose that all assumptions of Theorem 5.1 and condition (5.19) are satisfied. Then all solutions of the equations

$$
D y=a y(L y)^{ \pm m}
$$

are of the form

$$
y^{ \pm}=E x^{ \pm}, \quad \text { where } x^{ \pm} \text {are defined by (5.21). }
$$

Proof. Let $m \in \mathbb{N}$. Let $x=L y$. By (5.23), we have $y=E L y=E x$, $D x=D L y=y^{-1} D y=a(L y)^{m}=a x^{m}$. This means that $x$ is a solution of (5.21), hence it is of the form (5.21). We conclude that a solution $y=E x$ is of the form (5.24). A similar proof for $-m \in \mathbb{N}$.

Proposition 5.4. Suppose that all assumptions of Proposition 5.3 are satisfied and $R b \in \operatorname{dom} \Omega^{-1}$, where $b \in X$ is given. Then all solutions of the equation

$$
D x=a x L x+b x
$$

are of the form

$$
\begin{aligned}
x & =E w, \quad \text { where } \\
w & =(E R a) R[b E(-R a)]+z E R a, \quad z \in \operatorname{ker} D \text { arbitrary } .
\end{aligned}
$$

Proof. Write $w=L x$. Then $x=E w$ and, by (5.25), we have $D w=$ $x^{-1} D x=a L x+b=a w+b$. This and Proposition 5.3 together imply that $w$ is defined by (5.26).

Propositions 5.3 and 5.4 together imply

COROllary 5.5. Suppose that all assumptions of Proposition 5.3 are satisfied. Then the initial value problem for (5.25) with the initial condition $F x=x_{0}$ has the unique solution

$$
x=E w_{0}, \quad \text { where } \quad w_{0}=(E R a) R[b E(-R a)]+x_{0} E R a .
$$


Theorem 5.2. Suppose that $X \in L(D),(L, E) \in G[\Omega], a \in \operatorname{dom} \Omega, h$ is an invertible mapping of $X$ into itself such that

$R[a h(x)] \subset \operatorname{dom} \Omega \cap \operatorname{dom} \Omega^{-1} \quad$ for $R \in \mathcal{R}_{D}$ whenever $x \in \operatorname{dom} D$ and $F$ is an initial operator for $D$ corresponding to $R$.

(i) The equations

$$
D x=\operatorname{axh}(x)(L x)^{ \pm n} \quad(n \in \mathbb{N})
$$

have solutions $x$ if and only if for $n \neq-1$ the equations

$$
x=E^{2}\left\{\mp \frac{1}{n+1} L\{\mp(n+1) R[a h(x)]+z\}\right\}
$$

(respectively) have solutions for $a z \in \operatorname{ker} D$.

If $n=-1$ then the only solution of (5.28) is an $x \in \operatorname{ker} D$ (i.e. $x$ is a constant). In that case, $F x=x, F D x=0$.

(iii) If $x$ is a solution of (5.29) for $n \neq-1$ then the initial condition $F x=x_{0}\left(x_{0} \in \operatorname{ker} D\right)$ is satisfied if and only if

$$
z=\left(L x_{0}\right)^{ \pm(n+1)} \quad \text { provided that } x_{0} \neq 0
$$

(i.e. there is no solution such that $F x=0$ ).

(iv) If for $n \neq-1$, condition (5.30) is satisfied and $F$ is multiplicative then FDx is not well-determined.

Proof. Write $u=L x, \tilde{h}=a h E$. Then $x=E u$ and $\tilde{h} L=a h E L=a h$. This, (5.28) and our assumptions together imply that for $\pm n(n \in \mathbb{N})$

$$
\begin{aligned}
D u^{\mp(n+1)} & =\mp(n+1) u^{\mp n} D u=\mp D u=\mp(n+1)(L x)^{\mp n} D L x \\
& =\mp(n+1)(L x)^{\mp n} x^{-1} D x=\mp(n+1) a h(x)=\mp(n+1) a h E u \\
& =\mp(n+1) \tilde{h}(u) .
\end{aligned}
$$

If $n \neq-1$ (i.e. $n+1 \neq 0$ ) then

$$
E(\mp L u)=u^{\mp(n+1)}=\mp(n+1) R \tilde{h}(u)+z,
$$

where $z \in \operatorname{ker} D$, i.e.

$$
\mp(n+1) L u=L E[\mp(n+1) L u]=L[\mp(n+1) R \tilde{h}(u)+z] .
$$

This implies that

$$
\begin{aligned}
x & =E u=E^{2} L u=E^{2}\left\{\mp \frac{1}{n+1} L[\mp R \tilde{h}(u)+z]\right\} \\
& =E^{2}\left\{\mp \frac{1}{n+1} L[\mp(n+1) R[a h E(L x)+z]\}\right. \\
& =E^{2}\left\{\mp \frac{1}{n+1} \operatorname{Rah}(x)+z\right\} \quad \text { where } z \in \operatorname{ker} D .
\end{aligned}
$$

This means that (5.29) should be satisfied for a $z \in \operatorname{ker} D$. 
(ii) If $n=-1$ then we get $D u^{-1}=0$, which implies that $u^{-1}=\tilde{z} \in \operatorname{ker} D$ and $x=E L x=E u=E \tilde{z}^{-1}=z \in \operatorname{ker} D$, i.e. $x$ is a constant. Hence $F x=F z=z=x$. Then $D x=0$. So $F D x=0$.

(iii) Suppose that $n \neq-1, x^{ \pm}$satisfy (5.29) and the initial value conditions

$$
F^{ \pm} x=x_{0}^{ \pm}, \quad \text { where } x_{0}^{ \pm} \in \operatorname{ker} D \text { are given, }
$$

respectively. This means that there are $z \in \operatorname{ker} D$ such that

$$
\begin{aligned}
x_{0}^{ \pm} & =F x_{0}^{ \pm}=F E^{2}\left\{\mp \frac{1}{n+1} F L\{\mp(n+1) R[a h(x)]+\tilde{z}\}\right\} \\
& =E^{2}\left\{\mp \frac{1}{n+1} F L\{\mp(n+1) R[a h(x)]+\tilde{z}\}\right\} \\
& =E^{2}\left\{\mp \frac{1}{n+1} L\{\mp(n+1) F R[a h(x)]+F \tilde{z}\}\right\} \\
& =E^{2}\left[\mp \frac{1}{n+1} L \tilde{z}\right]=E^{2} L z^{\mp \frac{1}{n+1}}=E z^{\mp \frac{1}{n+1}},
\end{aligned}
$$

and $z=\left(L x_{0}\right)^{\mp(n+1)}$.

(iv) If (iii) is satisfied and $F$ is multiplicative then

$$
F D x=F\left[a h(x)(L x)^{n}\right]=(F a) \cdot 0 \cdot[F h(x)](L F x)^{n} .
$$

However, $L F x=L(0)$ is not well-determined, so neither is $F D x$.

ExAmple 5.4. Suppose that all assumptions of Theorem 5.2 are satisfied. Then $x \in \operatorname{dom} D$ is a solution of the equation

$$
D x=\operatorname{axh}(x)
$$

if and only if $x \in \operatorname{dom} D$ is a solution of the equation

$$
x=z E R[a h(x)] \quad \text { for a } z \in \operatorname{ker} D .
$$

Indeed, we have $D L x=x^{-1} D x=a x h(x)$. Hence $x=E L x=E\{R[a h(x)]+$ $L z\}=(E L z) E R[a h(x)]=z E R[a h(x)]$, where $z \in \operatorname{ker} D$. If $F$ is an almost averaging initial operator for $D$ corresponding to $R$ then the initial value condition $F x=x_{0}\left(x_{0} \in\right.$ ker $D$ is given $)$ implies that

$x_{0}=F\{z E R[a h(x)]\}=z F E R[a h(x)]=z E F R[a h(x)]=z E(0)=z \cdot e=z$, i.e. $x$ should satisfy the equation

$$
x=x_{0} E R[a h(x)] .
$$

EXAmple 5.5. Suppose that all assumptions of Theorem 5.2 are satisfied, $g=R e \in I(X), a=g^{-1}, R g^{-1} \in \operatorname{dom} \Omega$ and $h(x)=x(x-e)$. Observe that $x^{-1}+(x-e)^{-1}=2 x^{-1}(x-e)^{-1}=2[x(x-e)]^{-1}$ whenever $x, x-e \in I(X)$. 
As before, we conclude that the equation

$$
D x=2 g^{-1} x(x-e)
$$

has a solution $x \in \operatorname{dom} D$ if and only $x \in \operatorname{dom} D$ satisfies the equation

$$
x(x-e)=z g^{2} \quad \text { for a } z \in \operatorname{ker} D .
$$

In that case, if $F$ is multiplicative, then the initial condition $F x=x_{0}\left(x_{0} \in\right.$ ker $D$ is given) leads to the equality $x_{0}\left(x_{0}-e\right)=z F g^{2}=z(F g)^{2}=0$. This implies that either $x_{0}=0$ or $x_{0}=e\left[{ }^{3}\right)$. If $F x=e$, we find $x-e=$ $R D(x-e)=R D x$, i.e.

$x=R D x+e=\left[2 g^{-1} x(x-e)\right]+e=R\left[2 g^{-1} z g^{2}\right]+e+2 z R g=2 z R^{2} e=z g^{2}$. Therefore $e=F x=F\left(z g^{2}\right)=z(F g)^{2}=0$, which is a contradiction. Hence $F x \neq e$. So $F x=0$. By similar arguments, we find that $F D x$ is not well defined, i.e. there is no $z \in \operatorname{ker} D$ such that $F D x=z$. Hence equation (5.36) has no solutions.

EXAMPLE 5.6. Suppose that all assumptions of Theorem 5.2 are satisfied, $g=R e \in I(X), a=g^{-1}$ and $F$ is an almost averaging initial operator for $D$ corresponding to $R$. Then the equation

$$
D x=g^{-1} x h(x) L x
$$

has a solution $x \in \operatorname{dom} D$ if and only if the equation

$$
x=E\left\{z E R\left[g^{-1} h(x)\right]\right\}
$$

has a solution $x \in \operatorname{dom} D$ for some $z \in \operatorname{ker} D$. Indeed, write $u=L x$. Then $x=E u$ and we have $D u=D L x=x^{-1} D x=g^{-1} h(x) L x=g^{-1} h(E u) u$. Hence $D L u=u^{-1} D u=g^{-1} h(E u)$, which implies that for $z \in \operatorname{ker} D$,

$$
x=E^{2} L^{2} x=E^{2} L\left\{z E R\left[g^{-1} h(x)\right]\right\}=E\left\{z E R\left[g^{-1} h(x)\right] .\right.
$$

If $x$ is a solution of (5.38) satisfying the initial condition $F x=x_{0}$, where $x_{0} \in \operatorname{ker} D$, then

$$
\begin{aligned}
x_{0} & =F x F E\left\{z E R\left[g^{-1} h(x)\right]\right\}=E F\left\{z E R\left[g^{-1} h(x)\right]\right\} \\
& =E\left\{z E F R\left[g^{-1} h(x)\right]\right\}=E[z E(0)]=E(z \cdot e)=E z .
\end{aligned}
$$

Let $x_{0}=0$. Since there is no $z \in \operatorname{ker} D$ such that $E z=x_{0}=0$, we conclude that $F x$ is not well-determined, hence there is no solution belonging to dom $D$. As in previous examples, we can show that in this case $F D x$ is not well-determined either.

\section{References}

[PR1] D. Przeworska-Rolewicz, Algebraic Analysis, PWN-Polish Sci. Publ. and Reidel, Warszawa-Dordrecht, 1988.

$\left({ }^{3}\right)$ Note that in Leibniz algebras constants are not zero divisors (cf. [PR1]). 
[PR2] D. Przeworska-Rolewicz, A priori determined solutions of linear equations, Math. Japonica 44 (1996), 395-412.

[PR3] -, Logarithms and Antilogarithms. An Algebraic Analysis Approach, with apppendix by Z. Binderman, Kluwer, Dordrecht, 1998.

[PR4] -, Postmodern logarithmo-technia, Comput. Math. Appl. 41 (2001), 1143-1154.

[PR5] - Algebraic analysis in structures with Kaplansky-Jacobson property, Studia Math. 168 (2005), 165-168.

[PR6] - Some summations formulae in commutative Leibniz algebras with logarithms, Control Cybernet. 36 (2007), 841-857.

[PRvT] D. Przeworska-Rolewicz and H. von Trotha, Right inverses in D- $R$ algebras with unit, J. Integral Equations 3 (1981), 245-259.

[R1] J. S. Ritt, Differential Equations from the Algebraic Stand Point, Amer. Math. Soc, New York, 1932.

[R2] - On the singular solutions of algebraic differential equations, Ann. of Math. 37 (1936), 552-617.

[T] H. Triebel, Analysis and Mathematical Physics, Reidel, Dordrecht, 1985.

[vT] H. von Trotha, Structure properties of $D-R$ spaces, Dissertationes Math. 180 (1981).

D. Przeworska-Rolewicz

Institute of Mathematics

Polish Academy of Sciences

Śniadeckich 8

00-956 Warszawa, Poland

E-mail: rolewicz@impan.pl

Received 22.9.2008

and in final form 2.12.2009 
